\title{
The impact of nonlinear restoring forces acting on hinged elastic beams
}

\author{
Filippo Gazzola Raffaella Pavani
}

\begin{abstract}
We consider a hinged elastic beam subject to a nonlinear restoring force. We show that the equilibrium position of the beam under the action of a vertical load displays an oscillating profile. We compare the nonlinear model with the linear model and we study the dependence of the solution with respect to the physical parameters involved.
\end{abstract}

\section{Introduction}

Assume that a hinged beam, of finite length $2 R$, is subject to the restoring forces of a large number of nonlinear two-sided springs as in Figure 1.

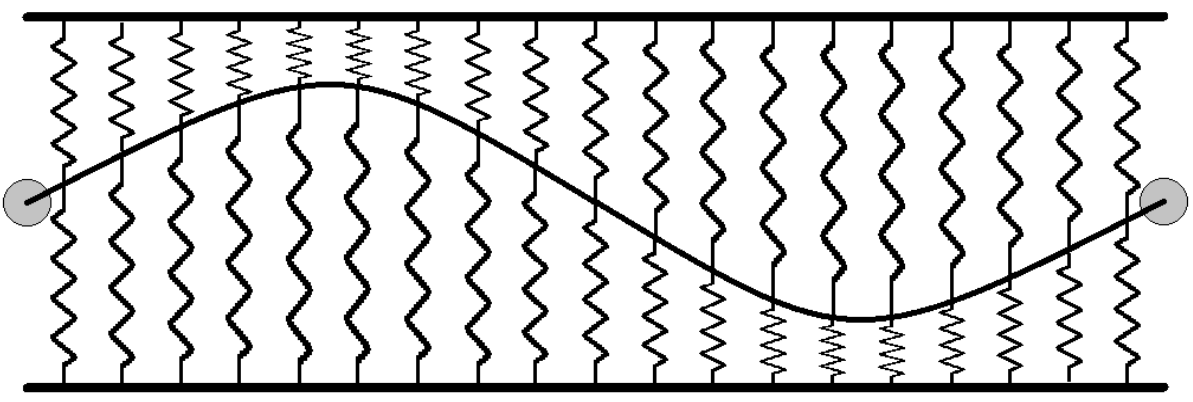

Figure 1: Beam subject to two-sided restoring springs.

Received by the editors in October 2014 - In revised form in February 2015.

Communicated by D. Bonheure.

2010 Mathematics Subject Classification : 34B15, 65L10, 74B20, 34C10.

Key words and phrases : hinged beam, nonlinear elasticity. 
The beam may be seen as a simplified model for the roadway of a suspension bridge and the springs are intended to model the hangers which, in turn, are linked to sustaining cables. Assume that, besides the nonlinear restoring force $g$ due to the springs there is a downwards distributed load $p=p(x)$ acting on the beam. Then, the vertical deformation $w$ of the beam is governed by the semilinear equation

$$
E I w^{\prime \prime \prime \prime}(x)-T w^{\prime \prime}(x)=p(x)-g(w(x)) \quad(-R<x<R)
$$

complemented with the hinged boundary conditions

$$
w( \pm R)=w^{\prime \prime}( \pm R)=0 .
$$

This is the general equation of a beam having flexural rigidity $E I>0$, constant tension $T \geq 0$, and subject to both a downwards load $p$ (its weight) and to the restoring action $g=g(w)$ due to some elastic springs; we refer to [2] (see also [6]) for the derivation of (1). We assume here that

$$
g \in C^{0}(\mathbb{R}) \text { is increasing. }
$$

It is well-known that the linear Hooke law of elasticity states that for relatively small deformations of an object, the size of the deformation is directly proportional to the deforming force or load. But for large values of the applied force, the deformation of the elastic material is often larger than expected on the basis of the Hooke law. This tells us that a linear law is reliable only for small elongations of a spring. In recent years, the nonlinear behavior of several structures became evident to the scientific community, see e.g. [12]; in particular, the nonlinear behavior of cables [10] and of the restoring action of the hangers [3] has been emphasized.

How different can be the response of a nonlinear restoring force was recently studied by us in several papers $[1,7,8,9]$ in the case of ideal infinite beams. A striking new phenomenon appears, namely a finite space blow up with selfexcited oscillation. More precisely, fourth order equations such as (1) exhibit solutions which cannot be continued to the whole real line due to the appearance of wide oscillations yielding $\lim \sup w(x)=+\infty$ and $\lim \inf w(x)=-\infty$ as $x$ approaches some finite limit position. We have shown that this phenomenon is not visible in equations of order less than four or in linear equations.

In this paper we aim at studying the same phenomenon in the case of a beam of finite length $2 R$, identified with the interval $[-R, R]$. We compare the difference between the solutions of the nonlinear problem (1)-(2) and the solutions of the corresponding linearized problem. We show that the profiles of the solutions of the nonlinear problem exhibit a larger number of oscillations and we study how this discrepancy varies in dependence of the physical parameters in the model.

Following a suggestion by Plaut-Davis [13, Section 3.5] we mainly consider

$$
g(w)=k w+\varepsilon w^{3} \text { for some } k, \varepsilon>0 ;
$$

in fact, this nonlinearity is quite common in elasticity models, see e.g. [11, (1)]. Let us also mention that, in view of the general results in [9], we expect different 
nonlinearities to generate the same qualitative behavior within solutions. In order to go directly into the heart of the matter, consider the initial value problem

$$
w_{\delta}^{\prime \prime \prime \prime}(x)-w_{\delta}^{\prime \prime}(x)+w_{\delta}(x)+\delta w_{\delta}(x)^{3}=1, \quad w_{\delta}(0)=w_{\delta}^{\prime}(0)=w_{\delta}^{\prime \prime}(0)=w_{\delta}^{\prime \prime \prime}(0)=0 .
$$

In Figure 2 we plot the solutions $w_{\delta}$ of (5) for $\delta=0$ (linear case), $\delta=0.01, \delta=0.02$. Close to $x=0$, say for $x \in[0,4.5]$, the solutions are almost identical. Then, the larger is $\delta$, the earlier the oscillation starts. For $\delta=0.02$ the solution numerically appears to blow up for $x \approx 7.1$.
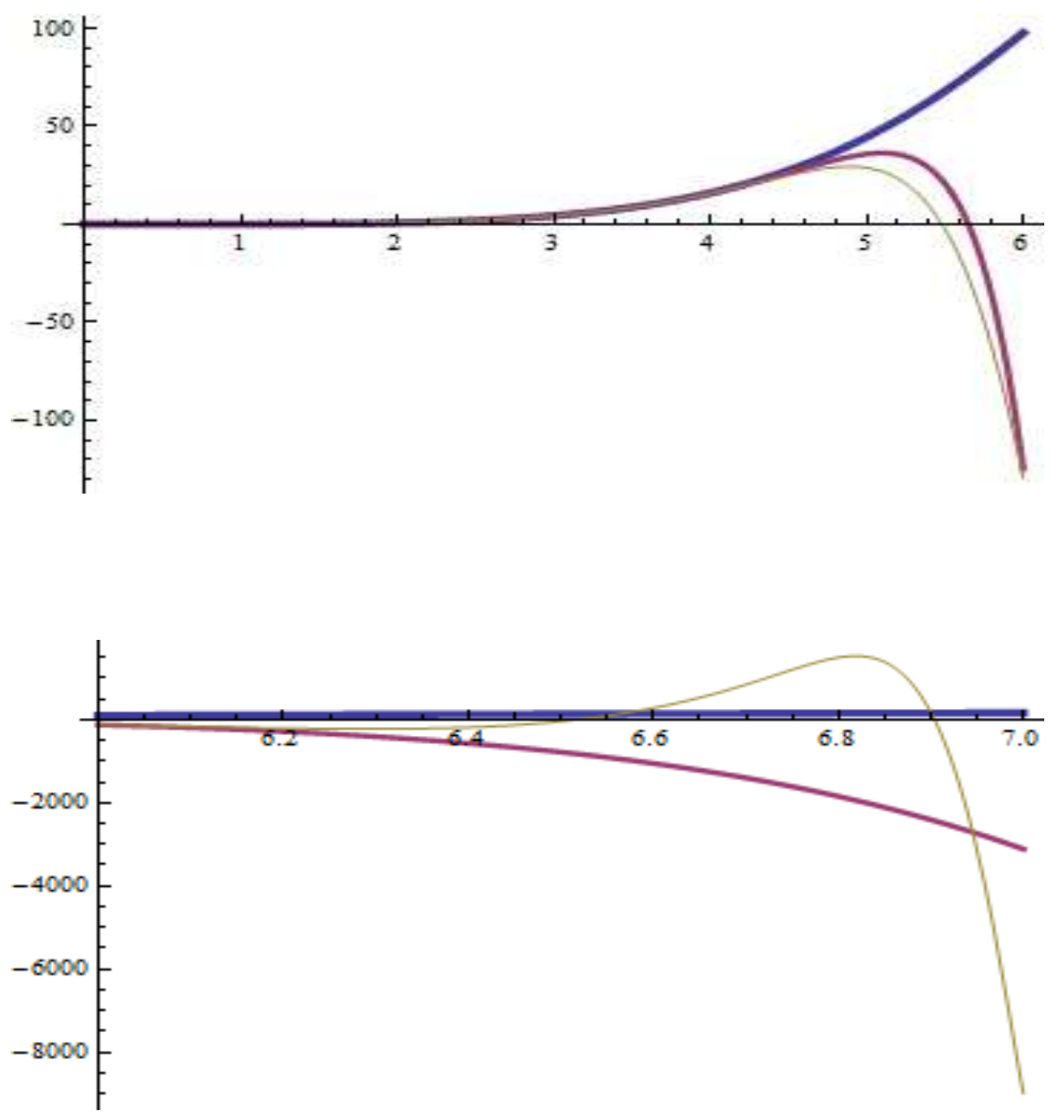

Figure 2: Solutions of (5) when $\delta=0$ (thick), $\delta=0.01$ (intermediate), $\delta=0.02$ (thin).

The purpose of this paper is to explain these behaviors and to emphasize the difference between the linear and the nonlinear regime in the case of a finite hinged beam subject to superlinear restoring forces. We will show that the presence of $\delta>0$ creates a phenomenon of self-excited oscillations which increases immeasurably the vibrations of the beam. As a conclusion, we may state that 
the nonlinear behavior of restoring forces creates fracture in ideal infinite beams whereas it increases the number of vibrations in hinged bounded beams.

\section{Preliminary results}

\subsection{Explicit solutions for the linear equation}

Consider first the linear case where $g(w)=k w$, that is, the springs obey the classical linear Hooke law. Then (1) reads

$$
E I w^{\prime \prime \prime \prime}(x)-T w^{\prime \prime}(x)+k w(x)=p(x) .
$$

The tension is usually small compared with the flexural rigidity; therefore, we assume that

$$
0 \leq T<2 \sqrt{k E I}
$$

This assumption gives the "right behavior" to the solutions of (6): indeed, assuming that $p$ is constant, $p(x) \equiv p \in \mathbb{R}$, simple calculus arguments show that if $T \geq 0$ satisfies (7), then the solutions of (6) have the form

$$
\begin{aligned}
& w(x)=a \cosh \left[x \sqrt{\frac{2 \sqrt{k E I}+T}{4 E I}}\right] \cos \left[x \sqrt{\frac{2 \sqrt{k E I}-T}{4 E I}}\right]+b \sinh \left[x \sqrt{\frac{2 \sqrt{k E I}+T}{4 E I}}\right] \sin \left[x \sqrt{\frac{2 \sqrt{k E I}-T}{4 E I}}\right] \\
& +c \sinh \left[x \sqrt{\frac{2 \sqrt{k E I}+T}{4 E I}}\right] \cos \left[x \sqrt{\frac{2 \sqrt{k E I}-T}{4 E I}}\right]+d \cosh \left[x \sqrt{\frac{2 \sqrt{k E I}+T}{4 E I}}\right] \sin \left[x \sqrt{\frac{2 \sqrt{k E I}-T}{4 E I}}\right]+\frac{p}{k}
\end{aligned}
$$

with the coefficients $a, b, c, d$ depending on the boundary or initial conditions. In particular, if we further assume that $w$ satisfies (2) then $w$ is even and it has the form

$$
w(x)=a \cosh \left[x \sqrt{\frac{2 \sqrt{k E I}+T}{4 E I}}\right] \cos \left[x \sqrt{\frac{2 \sqrt{k E I}-T}{4 E I}}\right]+b \sinh \left[x \sqrt{\frac{2 \sqrt{k E I}+T}{4 E I}}\right] \sin \left[x \sqrt{\frac{2 \sqrt{k E I}-T}{4 E I}}\right]+\frac{p}{k}
$$

for some $a, b \in \mathbb{R}$ depending on $R$. Too many parameters appear in the explicit form (8) of the solution. For this reason, in Section 2.3 we reduce the problem to an adimensional form.

\subsection{Variational formulation and well posedness of the nonlinear problem}

It is quite standard to prove that (1) is well-posed, it suffices to set up a suitable variational formulation. Let us briefly sketch what is meant by weak solution and under which assumptions it exists and is unique.

Consider the second order Sobolev space $H^{2} \cap H_{0}^{1}(-R, R)$ and let $H^{*}(-R, R)$ denote its dual space. The space $H^{2} \cap H_{0}^{1}(-R, R)$ is a Hilbert space when endowed with the scalar product

$$
(u, v)=\int_{-R}^{R} u^{\prime \prime}(x) v^{\prime \prime}(x) d x \quad \forall u, v \in H^{2} \cap H_{0}^{1}(-R, R) .
$$


For all $p \in H^{*}(-R, R)$ we say that $w \in H^{2} \cap H_{0}^{1}(-R, R)$ is a weak solution of (1)-(2) if

$$
\begin{aligned}
\int_{-R}^{R}\left(E I w^{\prime \prime}(x) v^{\prime \prime}(x)+T w^{\prime}(x) v^{\prime}(x)+g(w(x)) v(x)\right) d x & =\langle p, v\rangle \\
\forall v & \in H^{2} \cap H_{0}^{1}(-R, R)
\end{aligned}
$$

where $\langle\cdot, \cdot\rangle$ denotes the duality between $H^{*}(-R, R)$ and $H^{2} \cap H_{0}^{1}(-R, R)$. Since $H^{2} \cap H_{0}^{1}(-R, R) \subset C^{1}[-R, R]$, the integral $\int g(w) v$ makes sense. Weak solutions of (1)-(2), as defined in (9), are critical points of the energy functional

$$
\begin{array}{r}
J(u)=\frac{E I}{2} \int_{-R}^{R} u^{\prime \prime}(x)^{2} d x+\frac{T}{2} \int_{-R}^{R} u^{\prime}(x)^{2} d x+\int_{-R}^{R} G(u(x)) d x-\langle p, u\rangle \\
\forall u \in H^{2} \cap H_{0}^{1}(-R, R)
\end{array}
$$

where $G(s)=\int_{0}^{s} g(\sigma) d \sigma$. If $g$ satisfies (3) then $G \in C^{1}(\mathbb{R})$ is a convex function. Hence, the functional $J$ consists in the sum of two quadratic (convex) terms, of an additional convex term containing $G$, and of a linear term. Therefore, $J$ is convex and since it is continuous and coercive in $H^{2} \cap H_{0}^{1}(-R, R)$ it admits a unique critical point, its absolute minimum, which is a weak solution $w$ of (9). If we additionally assume that $p \in C^{0}[-R, R]$ (in particular, if $p$ is constant) then the weak solution $w$ belongs to $C^{4}[-R, R]$ and it is a classical solution of (1)-(2). Let us summarize these facts in the following

Theorem 1. Assume (3) and (7). Then for all $p \in H^{*}(-R, R)$ there exists a unique $w \in H^{2} \cap H_{0}^{1}(-R, R)$ satisfying (9). If $p \in C^{0}[-R, R]$ then $w \in C^{4}[-R, R]$ and $w$ is a classical solution of (1)-(2).

\subsection{Adimensional form of the equation}

With the choice (4), the equation (1) reads

$$
E I w^{\prime \prime \prime \prime}(x)-T w^{\prime \prime}(x)+k w(x)+\varepsilon w(x)^{3}=p(x) \quad(-R<x<R) .
$$

To simplify the task, we restrict our attention to the case of a constant load $p(x) \equiv p$. After the changes of variables

$$
x \mapsto x \sqrt[4]{\frac{k}{4 E I}}, \quad \tau=\frac{T}{2 \sqrt{k E I}}, \quad \delta=\frac{4 \varepsilon}{k}, \quad P=\frac{4}{k} p, \quad L=R \sqrt[4]{\frac{k}{4 E I}},
$$

this equation becomes

$$
w^{\prime \prime \prime \prime}(x)-4 \tau w^{\prime \prime}(x)+4 w(x)+\delta w(x)^{3}=P \quad(-L<x<L)
$$

with $\tau<1$ in view of (7). The boundary conditions (2) simply become

$$
w( \pm L)=w^{\prime \prime}( \pm L)=0 .
$$




\section{The number of oscillations of the solution of the linear problem}

When $\delta=0$, the equation (10) reads

$$
w^{\prime \prime \prime \prime}(x)-4 \tau w^{\prime \prime}(x)+4 w(x)=P \quad(-L<x<L)
$$

and the solution of (12) satisfying (11) is even for all $L>0$; by (8), the general solution of (12)-(11) is

$$
w(x)=a \cosh [x \sqrt{1+\tau}] \cos [x \sqrt{1-\tau}]+b \sinh [x \sqrt{1+\tau}] \sin [x \sqrt{1-\tau}]+\frac{P}{4}
$$

with $a$ and $b$ to be determined in dependence of $L$. For our convenience we restrict the possible values of $L$ by requiring that

$$
\frac{a}{\sqrt{1+\tau}}=\frac{b}{\sqrt{1-\tau}}=: \gamma
$$

in such a way that the solution reads

$$
\begin{aligned}
w(x)=\gamma\{\sqrt{1+\tau} \cosh [x \sqrt{1+\tau}] \cos [x \sqrt{1-\tau}] \\
+\sqrt{1-\tau} \sinh [x \sqrt{1+\tau}] \sin [x \sqrt{1-\tau}]\}+\frac{P}{4}
\end{aligned}
$$

By differentiating we then find that

$$
w^{\prime}(x)=2 \gamma \sinh [x \sqrt{1+\tau}] \cos [x \sqrt{1-\tau}]
$$

and it is therefore quite simple to compute the number of critical points of $w$. Restricting to the half-line $x \geq 0$, we see that $w^{\prime}(x)=0$ if and only if

$$
x=x_{0}:=0 \quad \text { or } \quad x=x_{j}:=\frac{(2 j-1) \pi}{2 \sqrt{1-\tau}} \quad(j \in \mathbb{N}, j \geq 1) .
$$

A further differentiation yields

$$
\begin{aligned}
w^{\prime \prime}(x)=2 \gamma\{\sqrt{1+\tau} \cosh [x \sqrt{1+\tau}] & \cos [x \sqrt{1-\tau}] \\
& -\sqrt{1-\tau} \sinh [x \sqrt{1+\tau}] \sin [x \sqrt{1-\tau}]\}
\end{aligned}
$$

By imposing the second boundary condition in (11) we find

$$
\tan [L \sqrt{1-\tau}]=\sqrt{\frac{1+\tau}{1-\tau}} \operatorname{coth}[L \sqrt{1+\tau}]
$$

and it is clear that

$$
\forall k \in \mathbb{N}(k \geq 1) \quad \exists ! L_{k} \in\left(\frac{(k-1) \pi}{\sqrt{1-\tau}}, \frac{(2 k-1) \pi}{2 \sqrt{1-\tau}}\right) \text { s.t. } L_{k} \text { satisfies (15). }
$$




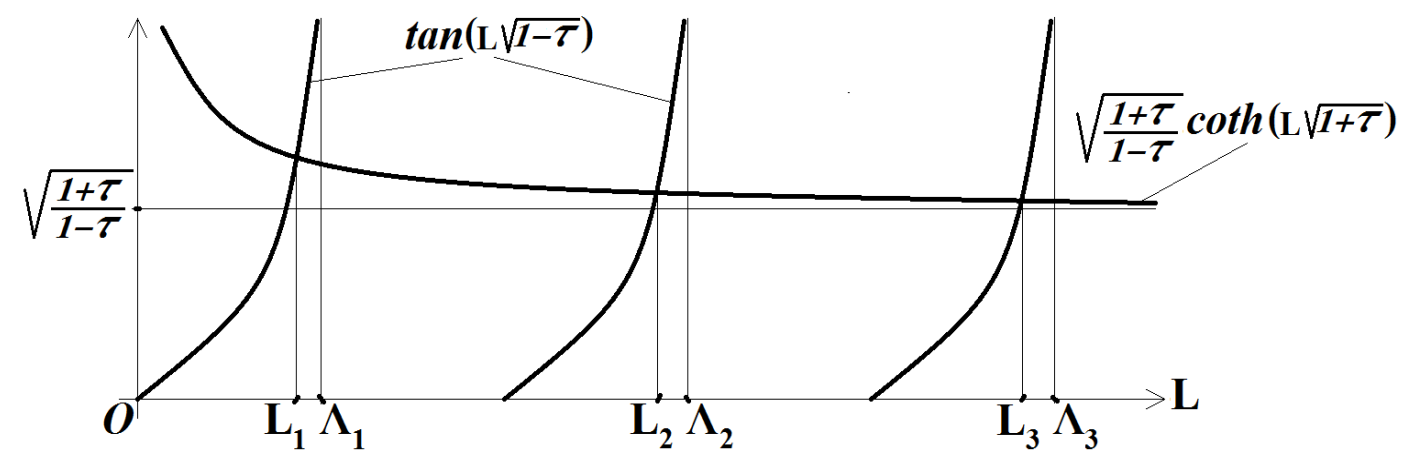

Figure 3: Positions of $L_{k}$ as defined in (16).

We refer to Figure 3, where $\Lambda_{k}=\frac{(2 k-1) \pi}{2 \sqrt{1-\tau}}$, for the qualitative description of the position of $L_{k}$ as defined in (16). In fact, since the right hand side of (15) is larger than 1 , we know that $L_{k}>\frac{(4 k-3) \pi}{4 \sqrt{1-\tau}}$.

Once $L_{k}$ is fixed we may compute $\gamma=\gamma_{k}$ by imposing the first boundary condition in (11) and by using (16):

$$
\gamma_{k}=-\frac{P}{8 \sqrt{1+\tau} \cosh \left[L_{k} \sqrt{1+\tau}\right] \cos \left[L_{k} \sqrt{1-\tau}\right]} .
$$

Since $L_{k}$ satisfies (16) we have $\gamma_{k}<0$.

Let us summarize the above results in the following statement.

Theorem 2. Assume that $L=L_{k}$ and $\gamma=\gamma_{k}$ for some $k \in \mathbb{N}(k \geq 1)$, where $L_{k}$ is defined in (16) and $\gamma_{k}$ is defined in (17). Then the function

$$
\begin{aligned}
w_{k}(x)=\gamma_{k}\{\sqrt{1+\tau} \cosh [x & \sqrt{1+\tau}] \cos [x \sqrt{1-\tau}] \\
& +\sqrt{1-\tau} \sinh [x \sqrt{1+\tau}] \sin [x \sqrt{1-\tau}]\}+\frac{P}{4}
\end{aligned}
$$

solves the problem

$w_{k}^{\prime \prime \prime \prime}(x)-4 \tau w_{k}^{\prime \prime}(x)+4 w_{k}(x)=P \quad \forall x \in\left(-L_{k}, L_{k}\right), \quad w_{k}\left( \pm L_{k}\right)=w_{k}^{\prime \prime}\left( \pm L_{k}\right)=0$.

Therefore, $w_{k}$ admits $2 k-1$ critical points given by $x_{0}$ and $\pm x_{j}$ for $j=1, \ldots, k-1$, see (14); in particular, if $k=1$ then $w_{1}$ only admits the unique critical point $x_{0}=0$.

Theorem 2 counts the number of critical points of $w_{k}$. Recalling that

the vertical axis is oriented downwards

we see that the unique constant solution of (12) is given by

$$
\widetilde{w}(x) \equiv \frac{P}{4} .
$$

Roughly speaking, we can say that the "target" of $w_{k}$, who aims to minimize the energy of the beam, is to approach as much as possible the equilibrium position 

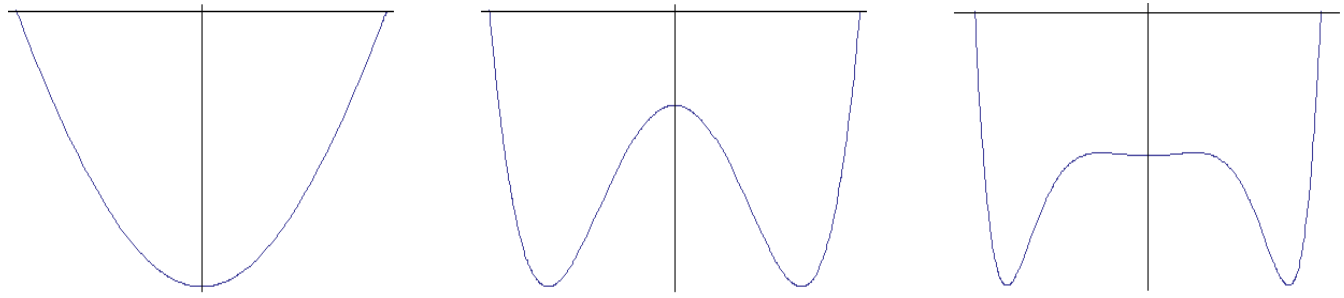

Figure 4: Graphs of $w_{1}, w_{2}, w_{3}$, as defined in Theorem 2.

$\widetilde{w}$. In Figure 4 we plot the qualitative graphs of $w_{1}, w_{2}, w_{3}$. One sees that the first beam is not large enough to allow $w_{1}$ to reach $P / 4$. On the other hand, the second beam is large enough and $w_{2}$ goes beyond $P / 4$ in order to get close to it after the first maximum point. Finally, the third beam tends to hide the oscillations of $w_{3}$ around $P / 4$; after the first maximum, $w_{3}$ just slightly oscillates around $P / 4$ and $w_{3}$ appears almost constant in the central part of the beam. The same phenomenon becomes more and more evident as $k$ increases, that is, as the length of the beam increases.

As a complement to Theorem 2 we may add two more information on the qualitative behavior of $w_{k}$. First, we may distinguish between maxima and minima points; we have

$$
\forall k \in \mathbb{N}, \quad k \geq 1 \quad(-1)^{k+j} w_{k}^{\prime \prime}\left(x_{j}\right)>0 \quad \forall j=0, \ldots, k-1 .
$$

Then, we formalize the tendency to flatten in the center of the beam as follows the map $\{0, \ldots, k-1\} \rightarrow \mathbb{R}_{+}$defined by $j \mapsto\left|w_{k}\left(x_{j}\right)-\frac{P}{4}\right|$ is strictly increasing.

Due to the linear nature of (12), it is clear that the number of critical points of $w_{k}$ does not depend on $P$. Moreover, we have linked $\tau$ to $L_{k}$ through (16). Therefore, the number of critical points of the solution $w_{k}$ of (12)-(11) (for $L=L_{k}$ ) merely depends on $k$. Theorem 2 states that it equals $2 k-1$. In next section we numerically compute the number of critical points of the solution of (10)-(11) and we compare it with $2 k-1$; however, for the nonlinear problem, the number of critical points depends both on $P$ and $\delta$.

\section{The number of oscillations of the solution of the nonlinear problem}

\subsection{The numerical procedure and its test on the linear equation}

In the nonlinear case $\delta \neq 0$ we do not have explicit solutions of (10)-(11) and we cannot proceed theoretically in order to find the number of critical points of the corresponding solutions. Let us notice that, by the Cardano formula, the unique 
constant solution of (10) is given by

$$
\begin{aligned}
\bar{w}(x) \equiv & W(\delta, P):= \\
& \frac{3 \cdot 4^{1 / 3} \cdot P}{\left[3 P \sqrt{3 \delta}+\sqrt{27 P^{2} \delta+256}\right]^{2 / 3}+4^{4 / 3}+\left[3 P \sqrt{3 \delta}-\sqrt{27 P^{2} \delta+256}\right]^{2 / 3}}
\end{aligned}
$$

which coincides with (18) when $\delta=0$.

Assume again that, for a given $\tau \in(0,1)$, the length of the beam is given by (16) for some $k \in \mathbb{N}(k \geq 1)$. We are interested in finding the number of critical points of the solution of (10)-(11) when $L=L_{k}$ and $\tau$ satisfies (16). By symmetry, we may restrict our attention to the interval $\left[0, L_{k}\right)$. This number also depends both on the load $P$ and on the nonlinear coefficient $\delta$; let us denote it by

$Z\left(L_{k}, P, \delta\right):=$ the number of critical points of the solution of (10)-(11) in the interval $\left[0, L_{k}\right)$.

Note that $Z\left(L_{k}, P, 0\right)=k$ for all $P$. By putting $w(x)=\alpha z(x)$, one sees that

$$
Z\left(L_{k}, P, \delta\right)=Z\left(L_{k}, \frac{P}{\alpha}, \delta \alpha^{2}\right) \quad \forall \alpha>0 .
$$

In particular, (23) states that the number of critical points does not vary if we decrease the nonlinearity and we increase the load (or viceversa) following a suitable rule.

In order to compute $Z\left(L_{k}, P, \delta\right)$ we proceeded numerically by using the bvptwp code, whose MATLAB version was published in [4]. It is an optimized highquality code. We first tested this numerical approach on the linear case $\delta=0$. We chose $\tau$ and $k$, then we computed $L_{k}$ as defined in (16): by a bisection method we found the root of $(15)$ in the interval $\left(\frac{(k-1) \pi}{\sqrt{1-\tau}}, \frac{(2 k-1) \pi}{2 \sqrt{1-\tau}}\right)$ with an accuracy of the order of $10^{-12}$. This choice is motivated by our numerical accuracy requirement. We note that any Newton (or Newton-type) method cannot be used since convergence is not guaranteed by the function behavior close to the searched root. Actually, the bvptwp code has an order which varies from 4 to 8; for the initial mesh we used a stepsize of the order of $10^{-3}$, and tolerances of about $10^{-10}$ so we can expect to compute the solution of (10) with about 10 correct digits.

We then compared the analytical results obtained in Section 3 with our numerical results. To this end, we first note that values of $\gamma_{k}$ in (17) decrease very quickly when $k$ increases; for example, $\gamma_{6}=4.2 \cdot 10^{-32}$ and $\gamma_{12}=8.8 \cdot 10^{-68}$ when $P=10$ and $\tau=0.9$. Therefore, the computation of $w_{k}(x)$ in Theorem 2 can exhibit a serious loss of significant digits due to the fact that the finite arithmetic sum can be an ill-conditioned operation. Indeed, if one wishes to compute the variations of the solution $w_{k}(x)$ (see Theorem 2 ) with respect to $\widetilde{w}(x)=P / 4$ (see $(18))$, one is led to introduce the function

$$
V(x)=w_{k}(x)-\frac{P}{4}=\left(\gamma_{k} A(x)+\frac{P}{4}\right)-\frac{P}{4}
$$

where $A(x)=\{\sqrt{1+\tau} \cosh [x \sqrt{1+\tau}] \cos [x \sqrt{1-\tau}]+\sqrt{1-\tau} \sinh [x \sqrt{1+\tau}]$ $\sin [x \sqrt{1-\tau}]\}$. 
We notice that, even though $V(x) \neq 0$ one may find $V(x)$ to be numerically zero : i) if $\gamma_{k} A(x)$ is less than the machine roundoff unit $\epsilon$; ii) given $P / A=m_{1} 2^{q}$ and $\gamma_{k} A(x)=m_{1} 2^{p}$, with $\left|m_{1}\right|,\left|m_{2}\right| \geq 1$, in a binary finite precision arithmetic, if the sum $\left(\gamma_{k} A(x)+P / 4\right)=\left(m_{2} 2^{p-q}+m_{1}\right) 2^{q}$ has the same finite representation of $P / 4$; this happens when $2^{p-q}<\epsilon$. To avoid this problem we computed $\gamma_{k} A(x)$ only; this enabled us to compute correct scaled critical values corresponding to the critical points given by (14). Whence, when we used the bvptwp code, we considered the function $z(x)=w(x)-P / 4$ so that, instead of (12), we consider the problem

$$
z^{\prime \prime \prime \prime}-4 \tau z^{\prime \prime}+4 z=0, \quad z( \pm L)=-\frac{P}{4}, \quad z^{\prime \prime}( \pm L)=0 .
$$

We consider here the case $k=12, \tau=0.9, P=10, L=L_{12}=113.535$. In Table 1 in the Appendix we quote the analytical critical points $x_{j}(j=1, \ldots, 12)$ given by (14), the numerically evaluated critical points $\xi_{j}$, the corresponding scaled critical values $V\left(x_{j}\right)$ (that is, $\left.\gamma_{k} A\left(x_{j}\right)\right)$ and $z\left(\tilde{\xi}_{j}\right)$, which were obtained solving numerically (25) by bvptwp. It appears clearly that the numerical critical values exhibit a very good accordance with the analytical ones; indeed, they often exhibit more than 7 correct digits. This was somehow expected since we have to consider the effect of both the error by numerical integration and the error in estimating the numerical critical points.

Critical points and critical values are computed as follows. We recall that the bvptwp code provides two vectors: the vector of the grid points built by dividing the integration interval $\left[-L_{k}, L_{k}\right]$ into $N$ subintervals, assuming the integration stepsize $h=2 L_{k} / N$, and $x_{n}=\left(\frac{2 n}{N}-1\right) L_{k}$ for $n=0,1, \ldots N$, together with the vector of the computed approximations $z_{n}$ of $z\left(x_{n}\right)$. Then we check if the origin is a local maximum or minimum. If the origin is a maximum, our routine looks for a local minimum, which is the first grid point where the solution value is followed by a larger value and then it goes on seeking a local maximum, which is the first grid point where the solution value is followed by a smaller value, and so on. The contrary happens when the origin is a local minimum. It is clear that for increasing $N$ and decreasing $h$, the error is decreasing. For Table 1 we chose $N=90000$ so that $h=0.00252$; therefore the absolute error in the estimated critical point $\xi_{j}$ is not greater than $3 \cdot 10^{-3}$. The corresponding values $z\left(\xi_{j}\right)$ may not be the exact critical values, they are computed at the closest point $\xi_{j}$ of the grid. We remark that we estimated critical points in $\left[0, L_{k}\right)$ only.

\subsection{Numerical results in the nonlinear case}

We consider here (10)-(11) with $\delta>0$. In order to compute the critical points and the critical values by the bvptwp code, we introduce the variable $z(x)=w(x)-$ $W(\delta, P)$ where $W(\delta, P)$ is defined in (21). Then, instead of (10)-(11), we consider the problem

$$
\begin{aligned}
z^{\prime \prime \prime \prime}-4 \tau z^{\prime \prime}+\left(4+3 W(\delta, P)^{2}\right) z+3 \delta W(\delta, P) z^{2}+\delta z^{3} & =0, \\
& z( \pm L)=-W(\delta, P), \quad z^{\prime \prime}( \pm L)=0 .
\end{aligned}
$$


If we fix $\delta=0.1, L=L_{12}=113.535(k=12)$, and we take $\tau=0.9$ and $P=10$, then we have $W(\delta, P)=2.224723963$ in (21). The computed critical points $\xi_{i}$ and corresponding critical values $z\left(\xi_{j}\right)$ are reported in Table 2 in the Appendix. In this case the origin is a maximum and

\section{the number of critical points is larger than in the linear case}

and, consequently, the last maximum, which is the absolute maximum, is closer to the right end of the integration interval. In order to check this behavior, we considered some different values of $\delta$ and $\tau$ whereas we always assumed $P=10$ in view of (23). The detailed results are contained in the Appendix. In Figure 5 we quote the plots of the map $Z\left(L_{k}, P, \delta\right)$ for different values of its arguments.
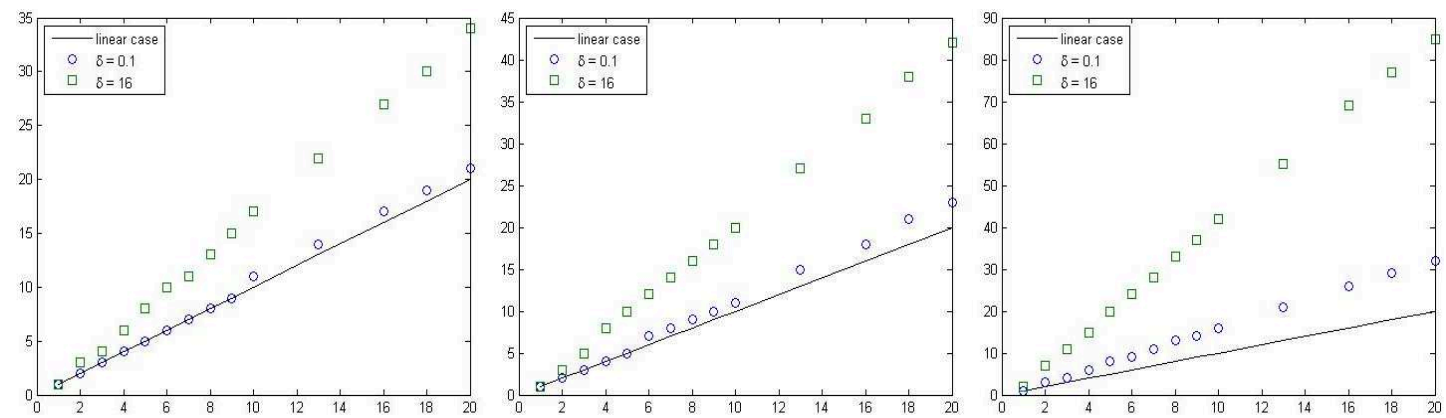

Figure 5: Plots of the maps $k \mapsto Z\left(L_{k}, 10, \delta\right)$ for $\delta=0,0.1,16$. The values of $\tau$ are $0.1,0.5,0.9$ from left to right.

It appears that the gap $Z\left(L_{k}, P, \delta\right)-k$ increases with $\tau$.

We also consider the two numbers

$$
\begin{aligned}
& M_{l}:=\max \left\{|z(x)| ; z^{\prime}(x)=0, z \text { solves }(25)\right\}, \\
& M_{n l}:=\max \left\{|z(x)| ; z^{\prime}(x)=0, z \text { solves }(26)\right\}
\end{aligned}
$$

which represent the maximum deviation of critical points with respect to the equilibrium positions (18) and (21).

If we compare Tables 3-4-5-6-7-8, and further numerical results that we obtained for different values of the parameters, we can draw the following conclusions:

- Tables 3-4, Tables 5-6, and Tables 7-8 suggest that

$$
\text { the } \operatorname{map} \delta \mapsto Z\left(L_{k}, P, \delta\right) \text { is increasing; }
$$

- in turn, by (23), this enables us to infer that

$$
\text { the map } P \mapsto Z\left(L_{k}, P, \delta\right) \text { is increasing; }
$$

- Tables 3-5-7 on one hand and Tables 4-6-8 on the other hand, suggest that

$$
\text { the map } \tau \mapsto Z\left(L_{k}, P, \delta\right) \text { is increasing; }
$$


- Tables 3-5-7 on one hand and Tables 4-6-8 on the other hand, suggest that

$$
\text { the maps } \tau \mapsto M_{l} \text { and } \tau \mapsto M_{n l} \text { are decreasing. }
$$

Moreover, it seems that $M_{l}>M_{n l}$ for small $\tau$ while $M_{l}<M_{n l}$ for large $\tau$.

Recalling the meaning of the parameters, we may conclude that a stronger nonlinearity of the restoring force, an increasing load, an increasing tension of the sustaining cable, all contribute to increase the number of vibrations within the beam. Moreover, the displacements from equilibrium $\left(M_{l}\right.$ and $\left.M_{n l}\right)$ are decreasing with respect to the tension $\tau$. Finally, the map $k \mapsto Z\left(L_{k}, P, \delta\right)-k$ is increasing; since $k$ somehow measures the length of the beam, as $k \rightarrow \infty$ we obtain the limit situation of an infinite number of oscillations, as shown in detail in our previous papers $[7,9]$.

\section{References}

[1] E. Berchio, A. Ferrero, F. Gazzola, P. Karageorgis, Qualitative behavior of global solutions to some nonlinear fourth order differential equations, J. Diff. Eq. 251, 2696-2727 (2011)

[2] M.A. Biot, T. von Kármán, Mathematical methods in engineering: an introduction to the mathematical treatment of engineering problems, XII, McGraw-Hill, New York (1940)

[3] J.M.W. Brownjohn, Observations on non-linear dynamic characteristics of suspension bridges, Earthquake Engineering \& Structural Dynamics 23, 1351-1367 (1994)

[4] J.R. Cash, D. Hollevoet, F. Mazzia, A.M. Nagy, Algorithm 927: The MATLAB code boptwp.m for the numerical solution of two point boundary value problems, ACM Transactions on Mathematical Software 39 (2), Article 15, (February 2013)

[5] F. Gazzola, Nonlinearity in oscillating bridges, Electron. J. Diff. Equ. no.211, 2013, 1-47

[6] F. Gazzola, H.-Ch. Grunau, G. Sweers, Polyharmonic boundary value problems, LNM 1991, Springer (2010)

[7] F. Gazzola, R. Pavani, Blow up oscillating solutions to some nonlinear fourth order differential equations, Nonlinear Analysis 74, 6696-6711 (2011)

[8] F. Gazzola, R. Pavani, Blow-up oscillating solutions to some nonlinear fourth order differential equations describing oscillations of suspension bridges, IABMAS12, $6^{\text {th }}$ International Conference on Bridge Maintenance, Safety, Management, Resilience and Sustainability, 3089-3093, Stresa 2012, Biondini \& Frangopol (Editors), Taylor \& Francis Group, London (2012) 
[9] F. Gazzola, R. Pavani, Wide oscillations finite time blow up for solutions to nonlinear fourth order differential equations, Arch. Rat. Mech. Anal. 207, 717-752 (2013)

[10] H.M. Irvine, Cable structures, MIT Press Series in Structural Mechanics, Massachusetts (1981)

[11] I.V. Ivanov, D.S. Velchev, M. Kneć, T. Sadowski, Computational models of laminated glass plate under transverse static loading, In: Shell-like structures, non-classical theories and applications; H. Altenbach, V. Eremeyev (Eds.), Springer, Berlin, Advanced Structured Materials 15, 469-490 (2011)

[12] W. Lacarbonara, Nonlinear structural mechanics, Springer (2013)

[13] R.H. Plaut, F.M. Davis, Sudden lateral asymmetry and torsional oscillations of section models of suspension bridges, J. Sound and Vibration 307, 894-905 (2007)

\section{Appendix: numerical tables}

Here we present the tables referred in Section 4 . Table 1 compares critical points $x_{j}$ of (25) (as given by (14)), rounded to 5 digits, with their numerical approximation $\xi_{j}$ and critical values $V\left(x_{j}\right)$ with their numerical approximations $z\left(\xi_{j}\right)$, when $k=12, \tau=0.9, P=10$.

\begin{tabular}{|c|c|c|c|}
\hline$x_{j}$ & $\xi_{j}$ & $V\left(x_{j}\right)$ & $z\left(\xi_{j}\right)$ \\
\hline 0 & 0 & $1.2100624 \mathrm{e}-67$ & $1.2100624 \mathrm{e}-67$ \\
\hline 4.9770 & 4.9780 & $1.3061401 \mathrm{e}-65$ & $1.3061399 \mathrm{e}-65$ \\
\hline 14.902 & 14.901 & $-1.1565620 \mathrm{e}-59$ & $-1.1565606 \mathrm{e}-59$ \\
\hline 24.836 & 24.836 & $1.0241123 \mathrm{e}-53$ & $1.0241123 \mathrm{e}-53$ \\
\hline 34.771 & 34.772 & $-9.0683073 \mathrm{e}-48$ & $-9.0683013 \mathrm{e}-48$ \\
\hline 44.706 & 44.705 & $8.0298027 \mathrm{e}-42$ & $8.0297981 \mathrm{e}-42$ \\
\hline
\end{tabular}

\begin{tabular}{|c|c|c|c|}
\hline$x_{j}$ & $\xi_{j}$ & $V\left(x_{j}\right)$ & $z\left(\xi_{j}\right)$ \\
\hline 54.640 & 54.640 & $-7.1102279 \mathrm{e}-36$ & $-7.1102276 \mathrm{e}-36$ \\
\hline 64.575 & 64.578 & $6.2959630 \mathrm{e}-30$ & $6.2959547 \mathrm{e}-30$ \\
\hline 74.509 & 74.509 & $-5.5749479 \mathrm{e}-24$ & $-5.5749470 \mathrm{e}-24$ \\
\hline 84.444 & 84.444 & $4.9365037 \mathrm{e}-18$ & $4.9365023 \mathrm{e}-18$ \\
\hline 94.378 & 94.377 & $-4.3711742 \mathrm{e}-12$ & $-4.3711695 \mathrm{e}-12$ \\
\hline 104.31 & 104.313 & $3.8705863 \mathrm{e}-6$ & $3.8705863 \mathrm{e}-6$ \\
\hline
\end{tabular}

Table 1: Comparison between the critical points (14) and their numerical approximation when $\delta=0, k=12, \tau=0.9, P=10$. 
Table 2 shows the computed values of the critical points $\xi_{j}$ of the solution of (10) together with their corresponding critical values $z\left(\xi_{j}\right)$, when $\delta=0.1, k=12$, $\tau=0.9, P=10$. Note that the number of critical points has increased from 12 to 19.

\begin{tabular}{|c|c|}
\hline$\xi_{j}$ & $z\left(\xi_{j}\right)$ \\
\hline 0 & $-1.749333299 \mathrm{e}-72$ \\
\hline 5.328 & $-3.063586089 \mathrm{e}-68$ \\
\hline 11.363 & $1.811528011 \mathrm{e}-64$ \\
\hline 17.398 & $-1.071173729 \mathrm{e}-60$ \\
\hline 23.433 & $6.333952062 \mathrm{e}-57$ \\
\hline 29.468 & $-3.745326049 \mathrm{e}-53$ \\
\hline 35.503 & $2.214646886 \mathrm{e}-49$ \\
\hline
\end{tabular}

\begin{tabular}{|c|c|}
\hline$\xi_{j}$ & $z\left(\xi_{j}\right)$ \\
\hline 41.538 & $-1.309541750 \mathrm{e}-45$ \\
\hline 47.573 & $7.743444819 \mathrm{e}-42$ \\
\hline 53.608 & $-4.578772508 \mathrm{e}-38$ \\
\hline 59.643 & $2.707471696 \mathrm{e}-34$ \\
\hline 65.678 & $-1.600953743 \mathrm{e}-30$ \\
\hline 71.713 & $9.466591617 \mathrm{e}-27$ \\
\hline
\end{tabular}

\begin{tabular}{|c|c|}
\hline$\xi_{j}$ & $z\left(\xi_{j}\right)$ \\
\hline 77.748 & $-5.597685581 \mathrm{e}-23$ \\
\hline 83.783 & $3.309964677 \mathrm{e}-19$ \\
\hline 89.818 & $-1.957213566 \mathrm{e}-15$ \\
\hline 95.853 & $1.157318971 \mathrm{e}-11$ \\
\hline 101.888 & $-6.843337144 \mathrm{e}-8$ \\
\hline 107.923 & $4.046373241 \mathrm{e}-4$ \\
\hline
\end{tabular}

Table 2: Numerical values of the critical points and critical values of the solution of (10) when $\delta=0.1, k=12, \tau=0.9, P=10$. 
We now consider different values of $\delta$ and $\tau$ whereas we keep $P=10$ fixed: by (23) the variations with respect to $P$ may be deduced by corresponding variations with respect to $\delta$.

Table 3 shows our results for $\delta=0.1, \tau=0.1, P=10$. The number $M_{l}$ denotes the absolute maximum of $V(x)$ as defined in (24), while $M_{n l}$ denotes the absolute maximum of the solution $z(x)$ of (26); in this case, we have $W(\delta, P)=2.224723963$ in (21). Finally, $Z\left(L_{k}\right)$ denotes the number of critical points of the solution of (10)-(11) in the interval $\left[0, L_{k}\right)$, see (22).

\begin{tabular}{|c|c|c|c|c|}
\hline$k$ & $L_{k}$ & $M_{l}$ & $Z\left(L_{k}\right)$ & $M_{n l}$ \\
\hline 1 & 1.00575 & -1.3426264 & 1 & -1.0779201 \\
\hline 2 & 4.19236 & $1.1419585 \mathrm{e}-1$ & 2 & $1.0047630 \mathrm{e}-1$ \\
\hline 3 & 7.50373 & $1.1786580 \mathrm{e}-1$ & 3 & $1.0290371 \mathrm{e}-1$ \\
\hline 4 & 10.8153 & $1.1786933 \mathrm{e}-1$ & 4 & $1.0290502 \mathrm{e}-1$ \\
\hline 5 & 14.1267 & $1.1786933 \mathrm{e}-1$ & 5 & $1.0290503 \mathrm{e}-1$ \\
\hline 6 & 17.4383 & $1.1786933 \mathrm{e}-1$ & 6 & $1.0290501 \mathrm{e}-1$ \\
\hline 7 & 20.7498 & $1.1786933 \mathrm{e}-1$ & 7 & $1.0290492 \mathrm{e}-1$ \\
\hline 8 & 24.0614 & $1.1786933 \mathrm{e}-1$ & 8 & $1.0290497 \mathrm{e}-1$ \\
\hline 10 & 30.6844 & $1.1786933 \mathrm{e}-1$ & 11 & $1.0290503 \mathrm{e}-1$ \\
\hline 13 & 40.6190 & $1.1786933 \mathrm{e}-1$ & 14 & $1.0290503 \mathrm{e}-1$ \\
\hline 16 & 50.5536 & $1.1786933 \mathrm{e}-1$ & 17 & $1.0290484 \mathrm{e}-1$ \\
\hline 20 & 63.9977 & $1.1786933 \mathrm{e}-1$ & 21 & $1.0290486 \mathrm{e}-1$ \\
\hline
\end{tabular}

Table 3: For varying $k$, absolute maximum and number of critical points of the solution of (10) when $\delta=0.1, \tau=0.1, P=10$. 
Table 4 shows our results for $\delta=16, \tau=0.1, P=10$. The number $M_{l}$ denotes the absolute maximum of $V(x)$ as defined in (24), while $M_{n l}$ denotes the absolute maximum of the solution $z(x)$ of (26); in this case, we have $W(\delta, P)=$ 0.7579901138 in (21). Again, $Z\left(L_{k}\right)$ denotes the number of critical points of the solution of (10)-(11) in the interval $\left[0, L_{k}\right)$, see (22). We also tried $\delta=60$ (with $\tau=0.1$ and $P=10$ ) which yields $W(\delta, P)=0.5100184968$ in (21): then for $k=20, \delta=60, \tau=0.1, P=10$, we found $Z\left(L_{k}\right)=38$ and $M_{n l}=0.0240770757$.

\begin{tabular}{|c|c|c|c|c|}
\hline$k$ & $L_{k}$ & $M_{l}$ & $Z\left(L_{k}\right)$ & $M_{n l}$ \\
\hline 1 & 1.00575 & -1.3426264 & 1 & $-3.3574287 \mathrm{e}-2$ \\
\hline 2 & 4.19236 & $1.1419585 \mathrm{e}-1$ & 3 & $3.5323444 \mathrm{e}-2$ \\
\hline 3 & 7.50373 & $1.1786580 \mathrm{e}-1$ & 4 & $3.5323677 \mathrm{e}-2$ \\
\hline 4 & 10.8153 & $1.1786933 \mathrm{e}-1$ & 6 & $3.5323676 \mathrm{e}-2$ \\
\hline 5 & 14.1267 & $1.1786933 \mathrm{e}-1$ & 8 & $3.5323670 \mathrm{e}-2$ \\
\hline 6 & 17.4383 & $1.1786933 \mathrm{e}-1$ & 10 & $3.5323635 \mathrm{e}-2$ \\
\hline 7 & 20.7498 & $1.1786933 \mathrm{e}-1$ & 11 & $3.5323667 \mathrm{e}-2$ \\
\hline 8 & 24.0614 & $1.1786933 \mathrm{e}-1$ & 13 & $3.5323658 \mathrm{e}-2$ \\
\hline 9 & 27.3729 & $1.1786933 \mathrm{e}-1$ & 15 & $3.5323613 \mathrm{e}-2$ \\
\hline 10 & 30.6844 & $1.1786933 \mathrm{e}-1$ & 17 & $3.5323637 \mathrm{e}-2$ \\
\hline 13 & 40.6190 & $1.1786933 \mathrm{e}-1$ & 22 & $3.5323656 \mathrm{e}-2$ \\
\hline 16 & 50.5536 & $1.1786933 \mathrm{e}-1$ & 27 & $3.5323534 \mathrm{e}-2$ \\
\hline 18 & 57.1767 & $1.1786933 \mathrm{e}-1$ & 30 & $3.5323645 \mathrm{e}-2$ \\
\hline 20 & 63.9977 & $1.1786933 \mathrm{e}-1$ & 34 & $3.5323553 \mathrm{e}-2$ \\
\hline
\end{tabular}

Table 4: For varying $k$, absolute maximum and number of critical points of the solution of (10) when $\delta=16, \tau=0.1, P=10$. 
Then we varied $\tau$. Table 5 shows our results for $\delta=0.1, \tau=0.5, P=10$. The number $M_{l}$ denotes the absolute maximum of $V(x)$ as defined in (24), while $M_{n l}$ denotes the absolute maximum of the solution $z(x)$ of (26); in this case, we have $W(\delta, P)=2.224723963$ in (21). Again, $Z\left(L_{k}\right)$ denotes the number of critical points of the solution of (10)-(11) in the interval $\left[0, L_{k}\right)$, see (22).

\begin{tabular}{|c|c|c|c|c|}
\hline$k$ & $L_{k}$ & $M_{l}$ & $Z\left(L_{k}\right)$ & $M_{n l}$ \\
\hline 1 & 1.51084 & $-7.9224393 \mathrm{e}-1$ & 1 & $-5.7092275 \mathrm{e}-1$ \\
\hline 2 & 5.92384 & $1.5423589 \mathrm{e}-2$ & 2 & $1.9924238 \mathrm{e}-2$ \\
\hline 3 & 10.3667 & $1.5490724 \mathrm{e}-2$ & 3 & $1.9961029 \mathrm{e}-2$ \\
\hline 4 & 14.8096 & $1.5490725 \mathrm{e}-2$ & 4 & $1.9961029 \mathrm{e}-2$ \\
\hline 5 & 19.2525 & $1.5490725 \mathrm{e}-2$ & 5 & $1.9961021 \mathrm{e}-2$ \\
\hline 6 & 23.0954 & $1.5490725 \mathrm{e}-2$ & 7 & $1.9961026 \mathrm{e}-2$ \\
\hline 7 & 28.1382 & $1.5490725 \mathrm{e}-2$ & 8 & $1.9961028 \mathrm{e}-2$ \\
\hline 8 & 32.5811 & $1.5490725 \mathrm{e}-2$ & 9 & $1.9961029 \mathrm{e}-2$ \\
\hline 9 & 37.0240 & $1.5490725 \mathrm{e}-2$ & 10 & $1.9961029 \mathrm{e}-2$ \\
\hline 10 & 41.4669 & $1.5490725 \mathrm{e}-2$ & 11 & $1.9961021 \mathrm{e}-2$ \\
\hline 13 & 54.7955 & $1.5490725 \mathrm{e}-2$ & 15 & $1.9961004 \mathrm{e}-2$ \\
\hline 16 & 68.1242 & $1.5490725 \mathrm{e}-2$ & 18 & $1.9961029 \mathrm{e}-2$ \\
\hline 18 & 77.0010 & $1.5490725 \mathrm{e}-2$ & 21 & $1.9961018 \mathrm{e}-2$ \\
\hline 20 & 85.8957 & $1.5490725 \mathrm{e}-2$ & 23 & $1.9961029 \mathrm{e}-2$ \\
\hline
\end{tabular}

Table 5: For varying $k$, absolute maximum and number of critical points of the solution of (10) when $\delta=0.1, \tau=0.5, P=10$. 
Table 6 shows our results for $\delta=16, \tau=0.5, P=10$. The number $M_{l}$ denotes the absolute maximum of $V(x)$ as defined in (24), while $M_{n l}$ denotes the absolute maximum of the solution $z(x)$ of (26); in this case, we have $W(\delta, P)=0.75799011$ in (21). Again, $Z\left(L_{k}\right)$ denotes the number of critical points of the solution of (10)(11) in the interval $\left[0, L_{k}\right)$, see (22).

\begin{tabular}{|c|c|c|c|c|}
\hline$k$ & $L_{k}$ & $M_{l}$ & $Z\left(L_{k}\right)$ & $M_{n l}$ \\
\hline 1 & 1.51084 & $-7.9224393 \mathrm{e}-1$ & 1 & $3.0910297 \mathrm{e}-2$ \\
\hline 2 & 5.92384 & $1.5423589 \mathrm{e}-2$ & 3 & $1.9736332 \mathrm{e}-2$ \\
\hline 3 & 10.3667 & $1.5490724 \mathrm{e}-2$ & 5 & $1.9736281 \mathrm{e}-2$ \\
\hline 4 & 14.8069 & $1.5490725 \mathrm{e}-2$ & 8 & $1.9736299 \mathrm{e}-2$ \\
\hline 5 & 19.2525 & $1.5490725 \mathrm{e}-2$ & 10 & $1.9736281 \mathrm{e}-2$ \\
\hline 6 & 23.6954 & $1.5490725 \mathrm{e}-2$ & 12 & $1.9736299 \mathrm{e}-2$ \\
\hline 7 & 28.1382 & $1.5490725 \mathrm{e}-2$ & 14 & $1.9736326 \mathrm{e}-2$ \\
\hline 8 & 32.5811 & $1.5490725 \mathrm{e}-2$ & 16 & $1.9736281 \mathrm{e}-2$ \\
\hline 9 & 37.0240 & $1.5490725 \mathrm{e}-2$ & 18 & $1.9736308 \mathrm{e}-2$ \\
\hline 10 & 41.4669 & $1.5490725 \mathrm{e}-2$ & 20 & $1.9736333 \mathrm{e}-2$ \\
\hline 13 & 54.7955 & $1.5490725 \mathrm{e}-2$ & 27 & $1.9736326 \mathrm{e}-2$ \\
\hline 16 & 68.1242 & $1.5490725 \mathrm{e}-2$ & 33 & $1.9736334 \mathrm{e}-2$ \\
\hline 18 & 77.0010 & $1.5490725 \mathrm{e}-2$ & 38 & $1.9736334 \mathrm{e}-2$ \\
\hline 20 & 85.8957 & $1.5490725 \mathrm{e}-2$ & 42 & $1.9736293 \mathrm{e}-2$ \\
\hline
\end{tabular}

Table 6: For varying $k$, absolute maximum and number of critical points of the solution of (10) when $\delta=16, \tau=0.5, P=10$. 
Table 7 shows our results for $\delta=0.1, \tau=0.9, P=10$. The number $M_{l}$ denotes the absolute maximum of $V(x)$ as defined in (24), while $M_{n l}$ denotes the absolute maximum of the solution $z(x)$ of $(26)$; in this case, we have $W(\delta, P)=2.224723963$ in (21). Again, $Z\left(L_{k}\right)$ denotes the number of critical points of the solution of (10)-(11) in the interval $\left[0, L_{k}\right)$, see (22).

\begin{tabular}{|c|c|c|c|c|}
\hline$k$ & $L_{k}$ & $M_{l}$ & $Z\left(L_{k}\right)$ & $M_{n l}$ \\
\hline 1 & 4.25417 & $-3.17518750 \mathrm{e}-2$ & 1 & $-5.8961180 \mathrm{e}-3$ \\
\hline 2 & 14.1887 & $3.8705820 \mathrm{e}-6$ & 3 & $4.0461082 \mathrm{e}-4$ \\
\hline 3 & 24.1233 & $3.8705863 \mathrm{e}-6$ & 4 & $4.0453454 \mathrm{e}-4$ \\
\hline 4 & 34.0579 & $3.8705863 \mathrm{e}-6$ & 6 & $4.0460479 \mathrm{e}-4$ \\
\hline 5 & 43.9925 & $3.8705863 \mathrm{e}-6$ & 8 & $4.0463708 \mathrm{e}-4$ \\
\hline 6 & 53.9271 & $3.8705863 \mathrm{e}-6$ & 9 & $4.0463704 \mathrm{e}-4$ \\
\hline 7 & 63.8616 & $3.8705863 \mathrm{e}-6$ & 11 & $4.0463733 \mathrm{e}-4$ \\
\hline 8 & 73.7962 & $3.8705863 \mathrm{e}-6$ & 13 & $4.0463504 \mathrm{e}-4$ \\
\hline 9 & 83.7309 & $3.8705863 \mathrm{e}-6$ & 14 & $4.0463656 \mathrm{e}-4$ \\
\hline 10 & 93.6654 & $3.8705863 \mathrm{e}-6$ & 16 & $4.0463711 \mathrm{e}-4$ \\
\hline 13 & 123.469 & $3.8705863 \mathrm{e}-6$ & 21 & $4.0463703 \mathrm{e}-4$ \\
\hline 16 & 153.273 & $3.8705863 \mathrm{e}-6$ & 26 & $4.0463634 \mathrm{e}-4$ \\
\hline 18 & 173.142 & $3.8705863 \mathrm{e}-6$ & 29 & $4.0463636 \mathrm{e}-4$ \\
\hline 20 & 193.011 & $3.8705863 \mathrm{e}-6$ & 32 & $4.0463622 \mathrm{e}-4$ \\
\hline
\end{tabular}

Table 7: For varying $k$, absolute maximum and number of critical points of the solution of (10) when $\delta=0.1, \tau=0.9, P=10$. 
Table 8 shows our results for $\delta=16, \tau=0.9, P=10$. The number $M_{l}$ denotes the absolute maximum of $V(x)$ as defined in (24), while $M_{n l}$ denotes the absolute maximum of the solution $z(x)$ of (26); in this case, we have $W(\delta, P)=$ 0.7579901138 in (21). Again, $Z\left(L_{k}\right)$ denotes the number of critical points of the solution of (10)-(11) in the interval $\left[0, L_{k}\right)$, see (22).

\begin{tabular}{|c|c|c|c|c|}
\hline$k$ & $L_{k}$ & $M_{l}$ & $Z\left(L_{k}\right)$ & $M_{n l}$ \\
\hline 1 & 4.25417 & $-3.17518750 \mathrm{e}-2$ & 2 & $9.7003992 \mathrm{e}-3$ \\
\hline 2 & 14.1887 & $3.8705820 \mathrm{e}-6$ & 7 & $9.6989325 \mathrm{e}-3$ \\
\hline 3 & 24.1233 & $3.8705863 \mathrm{e}-6$ & 11 & $9.6989375 \mathrm{e}-3$ \\
\hline 4 & 34.0579 & $3.8705863 \mathrm{e}-6$ & 15 & $9.6989332 \mathrm{e}-3$ \\
\hline 5 & 43.9925 & $3.8705863 \mathrm{e}-6$ & 20 & $9.6989375 \mathrm{e}-3$ \\
\hline 6 & 53.9271 & $3.8705863 \mathrm{e}-6$ & 24 & $9.6989130 \mathrm{e}-3$ \\
\hline 7 & 63.8616 & $3.8705863 \mathrm{e}-6$ & 28 & $9.6988994 \mathrm{e}-3$ \\
\hline 8 & 73.7962 & $3.8705863 \mathrm{e}-6$ & 33 & $9.6988819 \mathrm{e}-3$ \\
\hline 9 & 83.7309 & $3.8705863 \mathrm{e}-6$ & 37 & $9.6989361 \mathrm{e}-3$ \\
\hline 10 & 93.6654 & $3.8705863 \mathrm{e}-6$ & 42 & $9.6989292 \mathrm{e}-3$ \\
\hline 13 & 123.469 & $3.8705863 \mathrm{e}-6$ & 55 & $9.6989373 \mathrm{e}-3$ \\
\hline 16 & 153.273 & $3.8705863 \mathrm{e}-6$ & 69 & $9.6989275 \mathrm{e}-3$ \\
\hline 18 & 173.142 & $3.8705863 \mathrm{e}-6$ & 77 & $9.6988885 \mathrm{e}-3$ \\
\hline 20 & 193.011 & $3.8705863 \mathrm{e}-6$ & 85 & $9.6989346 \mathrm{e}-3$ \\
\hline
\end{tabular}

Table 8: For varying $k$, absolute maximum and number of critical points of the solution of (10) when $\delta=16, \tau=0.9, P=10$.

Dipartimento di Matematica del Politecnico,

Piazza L. da Vinci 32

20133 Milano (Italy)

emails: filippo.gazzola@polimi.it, raffaella.pavani@polimi.it 